\title{
Visible Light Communication Using DC-biased Optical Filter Bank Multi-carrier Modulation
}

\author{
Rui Chen*†, Ki-Hong Park*, Chao Shen ${ }^{\dagger}$, Tien Khee $\mathrm{Ng}^{\dagger}$, Boon S. Ooi ${ }^{\dagger}$ and Mohamed-Slim Alouini* \\ ${ }^{*}$ Communication Theory Laboratory, King Abdullah University of Science and Technology (KAUST) \\ Email: slim.alouini@kaust.edu.sa \\ ${ }^{\dagger}$ Photonics Laboratory, King Abdullah University of Science and Technology (KAUST) \\ Email: boon.ooi@kaust.edu.sa
}

\begin{abstract}
Filter bank multicarrier (FBMC) has become a promising candidate to replace conventional orthogonal frequency-division multiplexing (OFDM) scheme in $5 G$ technology due to its better spectral confinement which results in a reduced inter-channel interference (ICI). However, the viability of using FBMC in visible light communication has not been verified. In this work we present the first experimental validation of the DC-biased optical filter bank multicarrier (DCOFBMC) modulation scheme over a free-space optical channel. Under different receiving powers, up to three times bit error rate performance improvement has been achieved using DCOFBMC with different overlapping factors compared to that of conventional DCO-OFDM.
\end{abstract}

\section{INTRODUCTION}

Visible light communication (VLC) has drawn great attention of researchers due to its broad, unlicensed spectrum and the fact that the communication systems using visible light will neither cause health hazards nor electronic interferences [1].

Conventionally, DC-biased optical orthogonal frequencydivision multiplexing (DCO-OFDM) is used as the modulation scheme for VLC systems, where the encoding and decoding processes are achieved by performing inverse fast Fourier transform (IFFT) and fast Fourier transform (FFT) at the transmitter and the receiver, respectively [2], [3]. Due to its multicarrier (MC) nature, OFDM is highly resilient to frequency selective fading. Moreover, at the receiver end, due to the implementation of the cyclic prefix (CP), a simple one-tap equalization can be performed on each subcarrier to efficiently estimate the channel. However, the IFFT process of the OFDM system implies a rectangular window with the same OFDM symbol length being applied in the time-domain signal. The sinc-alike frequency response of the rectangular window has a very high side-lobe which is only about $13 d B$ lower than its main lobe (Fig. 1 and Fig. 2).

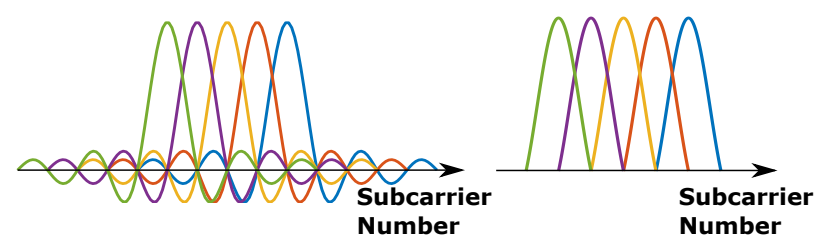

Fig. 1. Spectrum of OFDM (left) and FBMC (right) subchannels
In practical systems, when the orthogonality of sub-channels is lost due to interferences/noises, the energy leaked from one sub-channel could be high enough to make several subchannels unusable [4]. Moreover, the poor spectral confinement performance of the rectangular window makes OFDM highly sensitive to synchronization errors, either in time domain or in frequency domain [5].

To improve the spectral confinement performance of OFDM, one common approach is to use raised-cosine window or its modified versions to perform pulse-shaping for OFDM signals [6]. However, by comparing their frequency spectrum with that of the rectangular window, it is shown in Fig. 2 that the side-lobe attenuation improvements provided by these filters are barely marginal. On the contrary, it is clear in Fig. 2 that FBMC systems adopting Mirabbasi-Martin filters have superior side-lobe attenuation performances [4], [7]. With the increase of the overlapping factor $K$, theoretically its side-lobe attenuation performance will become even higher [4].

Research projects employing FBMC in radio frequency (RF) and fibre-optical communications have been reported in literature [8]-[10]. However, to the best of the authors' knowledge, the viability of using FBMC in VLC channel has not been experimentally verified.

Thus in this work, we studied the possibility of introducing DC-biased optical FBMC (DCO-FBMC) modulation scheme into free-space optical communication to effectively alleviate the spectral leakage problem and provide a better ICI protection which eventually leads to a better bit error rate (BER) performance. A comparison of the BER performance of DCOOFDM and DCO-FBMC with different overlapping factors are presented. A three times BER performance improvement has been observed in our laser-based VLC link using DCO-FBMC at $1.5 \mathrm{Gbit} / \mathrm{s}$. Our results are promising for the eventual realization of high performance laser diode based VLC system.

The rest of this paper will be organized as follows: section II will compare this work with other works in literature and point out the advances of this research. Section III will explain the experiment set-up of this work. Within different subsections, the design of the transmitter and the receiver will be explained in detail. The results and analysis will be presented in section IV while a final conclusion will be given in section V. 


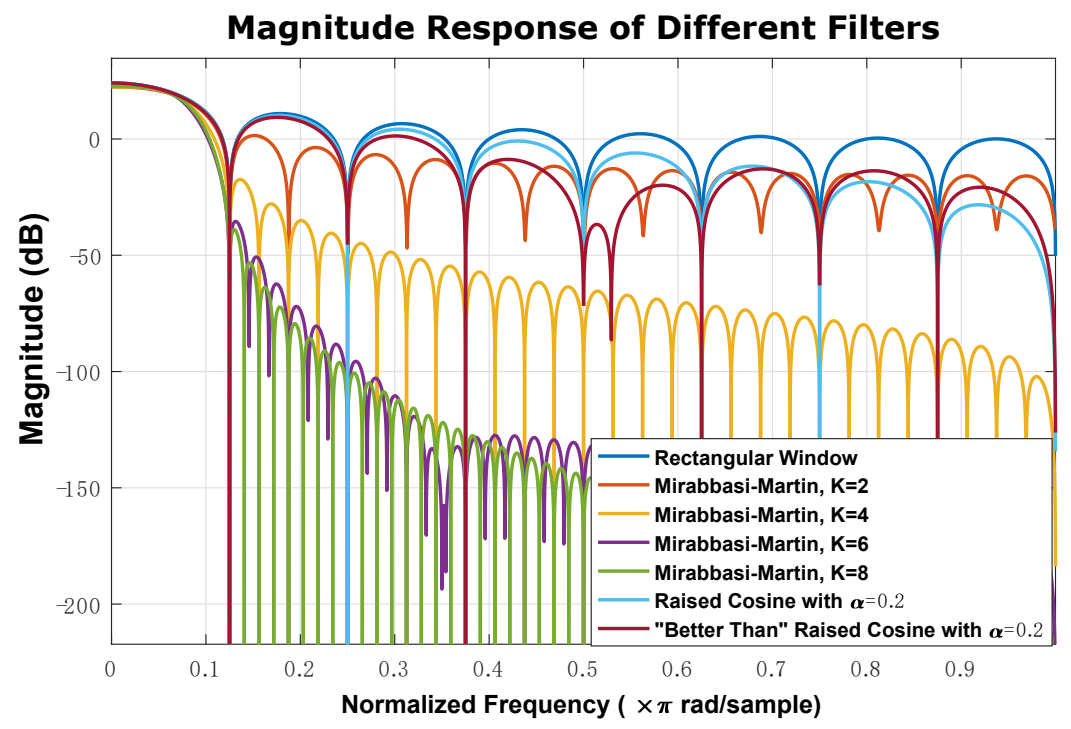

Fig. 2. A comparison of different window functions in frequency domain (IFFT size $=16$ ). Here the parameter $K$ is the overlapping factor for Mirabbasi-Martin filter. "Better Than" raised cosine window proposed in [6] with a roll-off factor $\alpha=0.2$ is added into comparison.

\section{RELATED WORKS}

OFDM based on offset quadrature amplitude modulation (OQAM-OFDM) is a particular type of FBMC scheme. This scheme has attracted a lot of attentions in RF wireless communication due to its superior spectral confinement performance and high resilience against synchronization errors when compared with the conventional OFDM scheme [5], [11][13]. However, to the best of our knowledge, its superiority compared with conventional DCO-OFDM in VLC channels has not been verified. Previous works such as [8] and [9] implemented OQAM-OFDM as a method to eliminate the use of CP and increase the spectral efficiency of the system. They focused on using either raised cosine or root-raised cosine filters, but the improvement on their side-lode attenuation performances is relatively minor (Fig. 2). The research by Jung et al. [14] did consider the implementation of Mirabbasi-Martin filter. However, their experiment was in fibre-optics, and the result is not directly applicable to VLC systems using intensity modulation/direct detection (IM/DD), which requires real and positive (intensity) signals to be applied. Later in 2016, Dang et al. [15] provided a simulation of FBMC in IM/DD channels. However, their results have not been verified by experiments. Moreover, they used a different $K$-channel cosine modulated filter bank (CMFB) to proceed the real-valued pulse amplitude modulation (PAM) symbols, while complex-valued quadrature amplitude modulation (QAM) symbols are more commonly used in OFDM systems [2], [3], [16].

Thus the advances of this research can be summarized as follows: (a) introduction of DCO-FBMC into VLC systems; (b) first known implementation of Mirabbasi-Martin filters in IM/DD channels; (c) validation of the superior spectral confinement performance and reduced ICI provided by DCOFBMC.

\section{EXPERIMENT SET-UP}

\section{A. Transmitter Design}

Fig. 3 shows the schematic for the experimental set-up. At the transmitter end, a pseudo-random binary sequence (PRBS) was generated in MATLAB ${ }^{\circledR}$. For the DCO-OFDM signal, after 64-QAM modulation, an IFFT of length 1024 was performed. During the subcarrier loading process shown in Fig. 4, Hermitian symmetry has to be applied to ensure purely real signal being generated.

After IFFT, a CP of 1 point was added to combat the intersymbol interference (ISI). The generated OFDM symbol in discrete time domain, can be denoted as:

$$
x(n)=\sum_{k=0}^{N-1} X(k) e^{\frac{j 2 \pi k n}{N}}
$$

where $X(k)$ is the frequency domain symbol, $k$ is the subcarrier index and $N$ is the IFFT length. The corresponding rectangular window, according to (1), can be denoted as:

$$
\begin{array}{r}
h(n)=\operatorname{rect}\left(\frac{n-N / 2}{N}\right) \\
\operatorname{rect}(n)= \begin{cases}1 & |n| \leq 1 / 2 \\
0 & \text { otherwise }\end{cases}
\end{array}
$$

and its frequency spectrum is shown in Fig. 2.

For the OQAM-OFDM signal, after QAM modulation and IFFT subcarrier loading, some extra procedures need to be taken:

- Each QAM symbol needs to be split into two parts, with real and imaginary parts interleaved as shown in Fig. 5.

- An up-sampling of $K$ times needs to be performed for each OQAM-OFDM symbol, where $K$ is defined as the overlapping factor of FBMC [7]. 


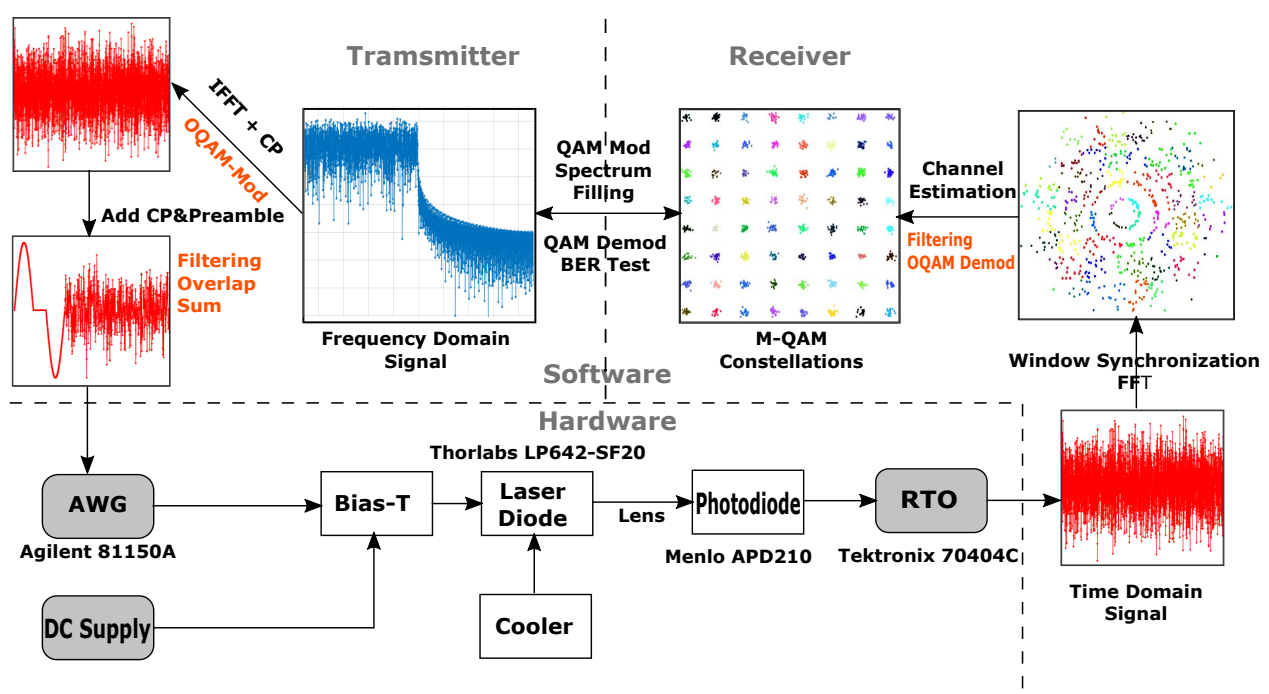

Fig. 3. System diagram of the designed system. Texts in orange denotes special processes required by OQAM-OFDM

\begin{tabular}{|c|c|c|c|c|c|c|c|c|c|}
\hline 0 & $x(2)$ & $x(3)$ & $X(4)$ & $\ldots$ & 0 & $\ldots$ & $\mathbf{X}(4) *$ & $x(3) *$ & $x(2) *$ \\
\hline $\begin{array}{r}1 \\
\text { Sub } \\
\text { Nun }\end{array}$ & $\underset{r}{\text { rier }} \stackrel{2}{a}$ & 3 & 4 & • & FT/ & 1 & N-2 & N-1 & $\mathbf{N}$ \\
\hline
\end{tabular}

Fig. 4. OFDM subcarrier loading scheme, where $X(k)$ denotes an individual QAM symbol, and $X(k)^{*}$ is the Hermitian transpose of $X(k)$

- Each OQAM-OFDM symbol was filtered, using the pulse-shaping filter defined in (3).

- An IFFT was performed, and individual time-domain OQAM-OFDM symbols were overlapped and summed with an interval of $N / 2$ (Fig. 6), where $N$ is again the IFFT length.

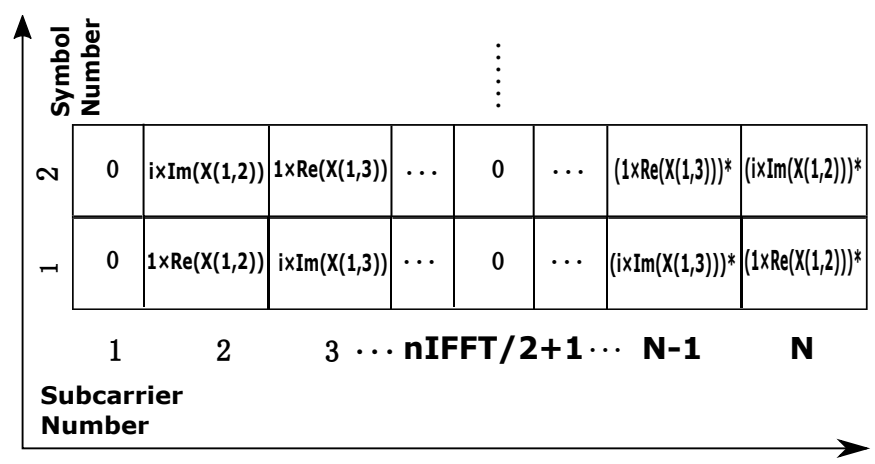

Fig. 5. OQAM-OFDM subcarrier loading scheme, where $X(m, k)$ denotes the QAM symbol for the $k$ th subcarrier in $m$ th OQAM-OFDM symbol, and $X(m, k)^{*}$ is the Hermitian transpose of $X(m, k)$

The Mirabbasi-Martin filter proposed in [4] with an overlapping factor $K$ of 2, 4, 6 and 8 was adopted, its time-domain impulse response can be denoted as:

$$
h(n)=1+2 \sum_{k=1}^{K-1}(-1)^{k} H_{k} \cos \left(2 \pi \frac{k n}{K N}\right)
$$

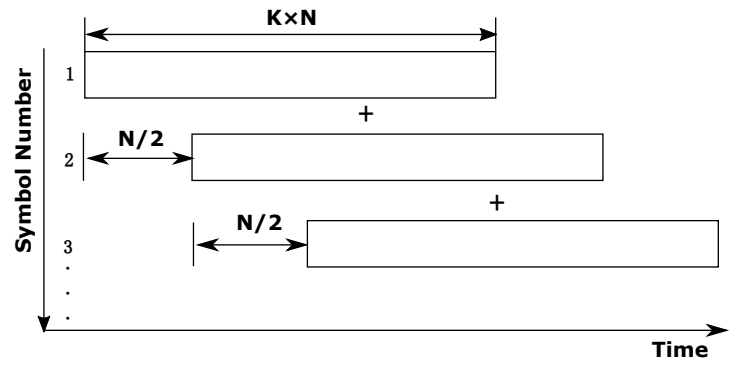

Fig. 6. OQAM-OFDM overlapping scheme in time domain

In this equation, $H_{k}$ is a set of coefficients related to $K$, which is introduced in [4].

As a result, the OQAM-OFDM signal in time domain can be denoted as [17]:

$$
\begin{aligned}
x(n)=\sum_{m=-\infty}^{+\infty} \sum_{k=0}^{N-1} A(m, k) h\left(n-m \frac{N}{2}\right) \\
\times e^{j 2 \pi / N k(n-(L-1) / 2)} e^{j \phi_{m, k}}
\end{aligned}
$$

where $L$ is the length of the pulse-shaping filter. And the definition of $A(m, k)$ and $\phi_{m, k}$ is:

$$
\begin{gathered}
A(2 m, 2 k)=\operatorname{Re}(X(m, 2 k)) \\
A(2 m, 2 k+1)=\operatorname{Im}(X(m, 2 k+1)) \\
A(2 m+1,2 k)=\operatorname{Im}(X(m, 2 k)) \\
A(2 m+1,2 k+1)=\operatorname{Re}(X(m, 2 k+1)) \\
\phi_{2 m, 2 k}=0 \quad \phi_{2 m, 2 k+1}=\pi / 2 \\
\phi_{2 m+1,2 k}=\pi / 2 \quad \phi_{2 m+1,2 k+1}=0
\end{gathered}
$$

As can be seen from the above processes, although in OQAM-OFDM scheme, each OFDM symbol needs to be split into two, the symbol rate doubles, resulting in a throughput that is the same as conventional DCO-OFDM. At the same 
time, as discussed above, the spectral confinement performance of OQAM-OFDM is much better than that of DCOOFDM, and increases with the $K$ value. This property can be clearly seen from the power spectral density (PSD) plots of both systems in Fig. 7.
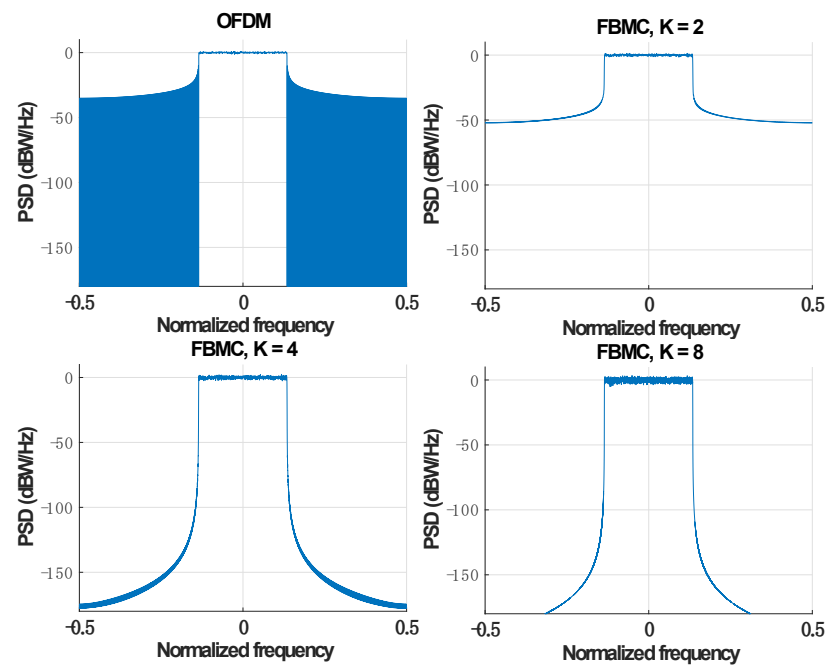

Fig. 7. Power spectral density of OFDM and FBMC with different $K$ values

To mitigate the intrinsic high peak to average power ratio problem of both DCO-OFDM and DCO-FBMC, a clipping has to be applied. Both the DCO-OFDM signal and the OQAM-OFDM signal were clipped at $3.6 \delta$, where $\delta$ is the standard variance of the signal. This clipping level was meticulously selected after exhaustive experiments to ensure a maximum signal to noise ratio (SNR) at the receiver, so that the highest possible BER performance could be achieved. After this, the discrete signal was downloaded to the Agilent Technologies 81150A $120 \mathrm{MHz}$ arbitrary waveform generator (AWG) with a sampling rate of 2 GSample/s. The generated signal was used to directly modulate a $642 \mathrm{~nm}$ red laser diode (LD, Thorlabs LP642-SF20). The optical power vs. current characteristics is measured as shown in Fig. 8. Thus, to ensure positive signal being generated, a DC bias of $94 m A$ was applied to the laser to provide a maximum usable linear range.

For proof-of-concept demonstration, the light was transmitted through a $20 \mathrm{~cm}$ transmission distance before received by the photo-detector (PD) while the attenuation effect for longdistance transmission was simulated by placing a set of neutral density filters between the LD and the PD.

\section{B. Receiver Design}

At the receiver end, the optical signal was detected by an avalanche photo-diode (Menlo Systems APD210) which was connected to a real-time oscilloscope (Tektronix 70404C). The signal was then downloaded to perform off-line analysis.

The window synchronization of the received signal is achieved by matched filtering [18]. At the transmitter, a specially designed preamble as in Fig. 9 was attached before the start of the signal.

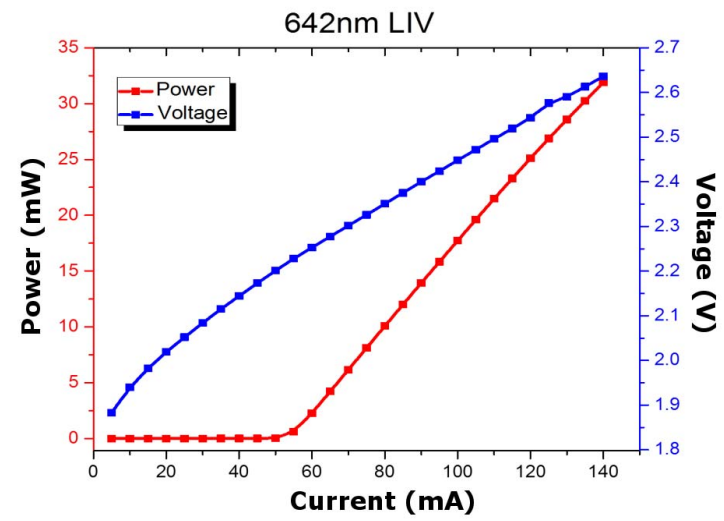

Fig. 8. Light-current-voltage (LIV) curve of the Thorlabs LP642-SF20 $642 \mathrm{~nm}$ red laser diode

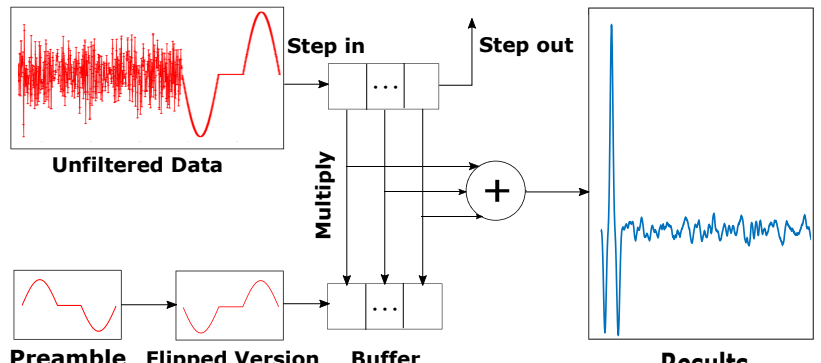

Fig. 9. Matched filtering process for start point detection

And at the receiver, the signal was sent into a matched filter with a template being the preamble. And the peak of the output was detected as the start point.

$$
\text { start }=\max _{k}\left(\sum_{n=1}^{N_{t}} t(n) x(n+k)\right)
$$

where $t(n)$ is the template, $N_{t}$ is the length of template and $k$ ranges from 0 to length $(x(n))-N_{t}+1$.

After the start point has been detected, for DCO-OFDM an FFT was performed after removing CP, followed by channel estimation and QAM demodulation. For DCO-FBMC, a matched filtering using the same pulse-shaping filter as in Eq. (3) was performed after FFT, followed by a down-sampling of $K$, channel estimation, OQAM demodulation and QAM demodulation.

The channel estimation scheme used in both systems is the preamble-based least square (LS) scheme. The only difference is that in the DCO-FBMC system, a prior knowledge of the pulse-shaping filter and first order neighbour subcarriers will be needed to calculate the intrinsic interference terms on individual subcarriers [19].

In LS scheme, a known sequence of pilot subcarriers $\boldsymbol{X}_{p}$ were transmitted, the channel response $\hat{\boldsymbol{h}}$ can be estimated using the received pilots $\boldsymbol{Y}_{\boldsymbol{p}}$, and the discrete Fourier transform 
matrix $\boldsymbol{F}$ as:

$$
\hat{\boldsymbol{h}}=\left(\boldsymbol{F}^{*} \boldsymbol{X}_{p}^{*} \boldsymbol{X}_{\boldsymbol{p}} \boldsymbol{F}\right)^{-1} \times \boldsymbol{F}^{*} \boldsymbol{X}_{p}^{*} \times \boldsymbol{Y}_{\boldsymbol{p}}
$$

After the decoding process, BER of different systems were derived for comparison. Due to limitations of components used, the $-3 d B$ bandwidth of the system is only around 250 $\mathrm{MHz}$ as shown in Fig. 10.

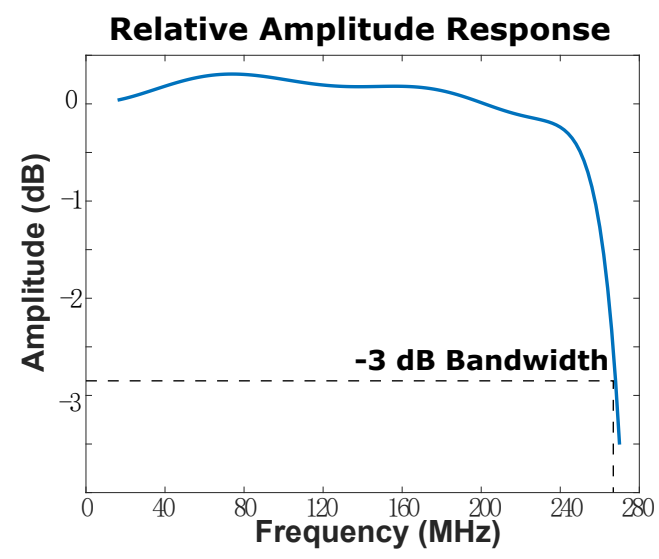

Fig. 10. Relative amplitude response of the system

The formula to calculate the transmission speed is given in equation (9), where $f_{s}$ is the sampling rate, $n$ is the number of used subcarriers, $M$ is the QAM order and $(N+C P)$ is the total symbol length.

$$
\text { Speed }=\frac{f_{s} \times n \times \log _{2}(M)}{(N+C P)}
$$

Hence, using 64-QAM, only 128 out of 1024 valid data subcarriers, and a 2 GSample/s sampling rate, the transmission speed was fixed at $1.5 \mathrm{Gbit} / \mathrm{s}$ for both DCO-OFDM and OQAM-OFDM for a fair comparison of their BER performance.

\section{Discussions}

Fig. 11(a) shows the BER performances of both DCOOFDM and OQAM-OFDM configurations at a received power from $-18 \mathrm{dBm}$ to $-3.5 \mathrm{dBm}$. A clearly enhanced BER performance was observed in OQAM-OFDM systems when the BER is lower than the forward error correction (FEC) limit of $3.8 \times 10^{-3}$, though there is insignificant improvement observed at a BER higher than FEC limit. Since the region where a received optical power of $>-7 \mathrm{dBm}$ is of particular interest, we did another experiment by reducing the channel distance to $5 \mathrm{~cm}$ and the result is shown in Fig. 11(b).

In consistence with what we see in Fig. 11(a), it is clear that OQAM-OFDM system exhibits a better BER performance than the DCO-OFDM system. For example, the BER was reduced from $1.24 \times 10^{-3}$ for DCO-OFDM to $4.1 \times 10^{-4}$ for OQAM-OFDM with $K=8$ at $0 \mathrm{dBm}$. With the increase of the overlapping factor $K$, this improvement becomes more obvious.

We attribute this observation to the better spectrum confinement provided by the scheme with a larger $K$ value.
In OQAM-OFDM, $K$ denotes the number of symbols that overlap in the time domain. When $K$ becomes larger, the outof-band attenuation provided by the filter also goes higher, leading to a much better spectrum confinement [7]. In real channels where interference/noise exists, this will significantly reduce possible ICI between adjacent subchannels. Moreover, the pulse-shaping filter implemented in FBMC is much better localized, both in time and in frequency domain as in Fig. 1, making the FBMC system more robust against possible synchronization errors, such as carrier frequency offset (CFO) and sampling frequency offset (SFO) which widely exist in OFDM systems [5], [13]. All of those factors contribute to the improved BER performance observed.

It is also noticed, however, that the BER performance of the FBMC system does not increase very significantly with a larger $K$ value. This might be due to the fact that the received FBMC symbols contain interference terms from neighbouring symbols in time/frequency domain [20]-[22]. In ideal channel conditions, these interference terms will only appear on the unused real/imaginary parts of individual subcarriers. But in practical channels, intrinsic interferences might introduce ISI/ICI into the system, causing a degraded BER performance. Thus with appropriate intrinsic interference cancellation schemes applied in further research, it is expected that the BER performance of DCO-FBMC system will become even better.

As for the computational complexity, DCO-FBMC has a higher computational complexity than DCO-OFDM mainly due to the increased FFT size and additional filtering processes. And this will increase with a larger $K$ value. However, this problem can be efficiently mitigated using the polyphase network (PPN) technique proposed in [7]. As the best tradeoff between performance and computational complexity, we found that $K=4$ should be used, which is consistent with the theoretical analysis [7], [15].

\section{CONCLUSION}

In summary, we firstly reported the transmission of OQAMOFDM over a VLC channel. The system uses a commercially available $642 \mathrm{~nm}$ red LD as the transmitter and an avalanche photodetector as the receiver. The BER performance of DCO-OFDM and OQAM-OFDM system under different attenuation levels are compared. At $1.5 \mathrm{Gbit} / \mathrm{s}$, the OQAMOFDM exhibits a superior performance over DCO-OFDM. This performance improvement becomes more obvious with a larger overlapping factor $K$. This study suggests that DCOFBMC has a great potential for future high-speed optical wireless communication systems.

\section{ACKNOWLEDGMENT}

The authors would like to acknowledge the financial support from KAUST-KFUPM Special Initiative, Grant No. REP/1/2878-01-01. 
(a)

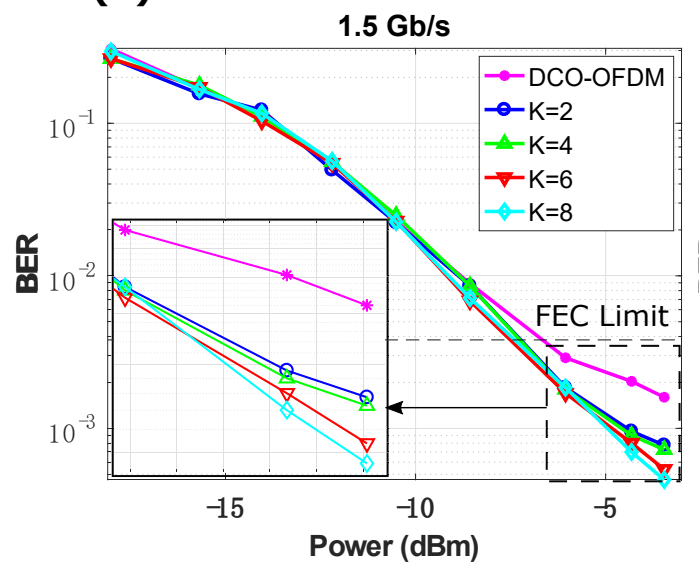

(b)

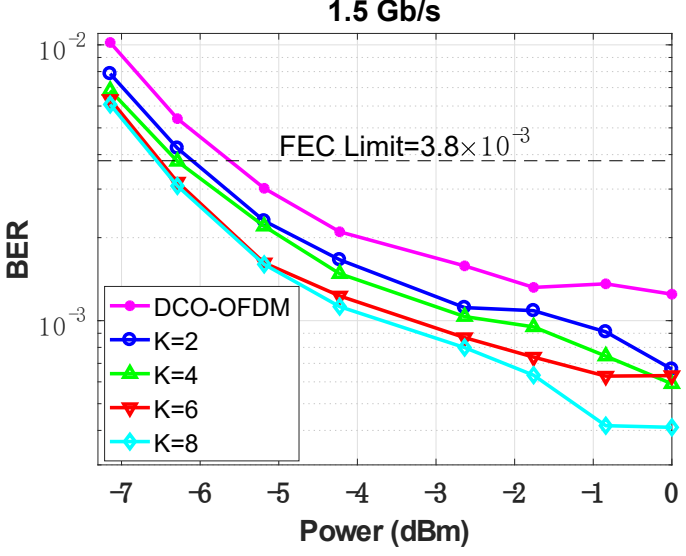

Fig. 11. BER performance of DCO-OFDM and OQAM-OFDM $(K=2,4,6,8)$ under different attenuation levels: (a) received power from $-18 d B m$ to $-3.5 \mathrm{dBm}$; (b) received power from $-7.1 \mathrm{dBm}$ to $0 \mathrm{dBm}$

\section{REFERENCES}

[1] M. Z. Afgani, H. Haas, H. Elgala, and D. Knipp, "Visible light communication using OFDM," in Testbeds and Research Infrastructures for the Development of Networks and Communities, 2006. TRIDENTCOM 2006. 2nd International Conference on. IEEE, 2006, pp. 6 pp.-134.

[2] D. Tsonev, H. Chun, S. Rajbhandari, J. J. D. McKendry, S. Videv, E. Gu, M. Haji, S. Watson, A. E. Kelly, G. Faulkner, M. D. Dawson, H. Haas, and D. O'Brien, "A 3-Gb/s single-LED OFDM-based wireless VLC link using a gallium nitride LED," IEEE Photonics Technology Letters, vol. 26, no. 7, pp. 637-640, 2014.

[3] B. Janjua, H. M. Oubei, J. R. D. Retamal, T. K. Ng, C.-T. Tsai, H.-Y. Wang, Y.-C. Chi, H.-C. Kuo, G.-R. Lin, J.-H. He, and B. S. Ooi, "Going beyond 4 Gbps data rate by employing RGB laser diodes for visible light communication," Optics Express, vol. 23, no. 14, pp. 18746-18753, 2015.

[4] S. Mirabbasi and K. Martin, "Overlapped complex-modulated transmultiplexer filters with simplified design and superior stopbands," IEEE Transactions on Circuits and Systems II: Analog and Digital Signal Processing, vol. 50, no. 8, pp. 456-469, 2003.

[5] H. Saeedi-Sourck, Y. Wu, J. W. M. Bergmans, S. Sadri, and B. FarhangBoroujeny, "Sensitivity analysis of offset QAM multicarrier systems to residual carrier frequency and timing offsets," Signal Processing, vol. 91, no. 7, pp. 1604-1612, 2011.

[6] P. Tan and N. C. Beaulieu, "Reduced ICI in OFDM systems using the "better than" raised-cosine pulse," IEEE Communications Letters, vol. 8, no. 3, pp. 135-137, 2004.

[7] M. Bellanger, D. Le Ruyet, D. Roviras, M. Terré, J. Nossek, L. Baltar, Q. Bai, D. Waldhauser, M. Renfors, and T. Ihalainen, "FBMC physical layer: A primer," PHYDYAS, January, 2010. [Online]. Available: http://www.ict-phydyas.org/teamspace/internal-folder/FBMCPrimer_06-2010.pdf

[8] F. Horlin, J. Fickers, P. Emplit, A. Bourdoux, and J. Louveaux, "Dualpolarization OFDM-OQAM for communications over optical fibers with coherent detection," Optics Express, vol. 21, no. 5, pp. 6409-6421, 2013.

[9] S. Randel, A. Sierra, X. Liu, S. Chandrasekhar, and P. J. Winzer, "Study of multicarrier Offset-QAM for spectrally efficient coherent optical communications," in 2011 37th European Conference and Exhibition on Optical Communication, Sept 2011, pp. 1-3.

[10] M. Renfors, P. Siohan, B. Farhang-Boroujeny, and F. Bader, "Filter banks for next generation multicarrier wireless communications," EURASIP Journal on Advances in Signal Processing, vol. 2010, no. 1, p. 314193, 2010.

[11] G. Wunder, P. Jung, M. Kasparick, T. Wild, F. Schaich, Y. Chen, S. T. Brink, I. Gaspar, N. Michailow, A. Festag, L. Mendes, N. Cassiau, D. Ktenas, M. Dryjanski, S. Pietrzyk, B. Eged, P. Vago, and F. Wiedmann, "5GNOW: non-orthogonal, asynchronous waveforms for future mobile applications," IEEE Communications Magazine, vol. 52, no. 2, pp. 97-105, February 2014.
[12] A. Sahin, I. Guvenc, and H. Arslan, "A survey on multicarrier communications: Prototype filters, lattice structures, and implementation aspects," IEEE Communications Surveys and Tutorials, vol. 16, no. 3, pp. 13121338, 2014.

[13] E. Kofidis, D. Katselis, A. Rontogiannis, and S. Theodoridis, "Preamblebased channel estimation in OFDM/OQAM systems: A review," Signal Processing, vol. 93, no. 7, pp. 2038-2054, 2013.

[14] S. Y. Jung, S. M. Jung, and S. K. Han, "AMO-FBMC for asynchronous heterogeneous signal integrated optical transmission," IEEE Photonics Technology Letters, vol. 27, no. 2, pp. 133-136, 2015.

[15] J. Dang, Z. Zhang, L. Wu, and L. Guo, "DC and non-DC biased optical filter bank multicarrier communication for IM/DD channel," in 2016 IEEE International Conference on Communications Workshops (ICC), May 2016, pp. 423-429.

[16] J. R. D. Retamal, H. M. Oubei, B. Janjua, Y.-C. Chi, H.-Y. Wang, C.-T. Tsai, T. K. Ng, D.-H. Hsieh, H.-C. Kuo, M.-S. Alouini, J.-H. He, G.-R. Lin, and B. S. Ooi, "4-Gbit/s visible light communication link based on 16-QAM OFDM transmission over remote phosphor-film converted white light by using blue laser diode," Opt. Express, vol. 23, no. 26, pp. $33656-33$ 666, 2015.

[17] P. Siohan, C. Siclet, and N. Lacaille, "Analysis and design of OFDM/OQAM systems based on filterbank theory," IEEE transactions on signal processing, vol. 50, no. 5, pp. 1170-1183, 2002.

[18] G. Turin, "An introduction to matched filters," IRE transactions on Information theory, vol. 6, no. 3, pp. 311-329, 1960.

[19] J. P. Javaudin, D. Lacroix, and A. Rouxel, "Pilot-aided channel estimation for OFDM/OQAM," in The 57th IEEE Semiannual Vehicular Technology Conference, 2003. VTC 2003-Spring., vol. 3, April 2003, pp. 1581-1585 vol.3.

[20] R. Zakaria and D. L. Ruyet, "A novel filter-bank multicarrier scheme to mitigate the intrinsic interference: Application to mimo systems," IEEE Transactions on Wireless Communications, vol. 11, no. 3, pp. 1112 1123, March 2012.

[21] _ , "Intrinsic interference reduction in a filter bank-based multicarrier using QAM modulation,” Physical Communication, vol. 11, pp. 15 - 24, 2014, Radio Access Beyond OFDM(A).

[22] D. Petrov, T. Haemaelaeinen, and S. Melnik, "Asymptotic presentation of intrinsic interference for fbmc/oqam signals in quasi-static channels," in European Wireless 2016; 22th European Wireless Conference, May 2016, pp. 1-6. 